\title{
Conformational isomerism in methyl cyanoacetate: A combined matrix-isolation infrared spectroscopy and molecular orbital study $\dagger$
}

\author{
Igor D. Reva, ${ }^{a}$ Sonia V. Ilieva ${ }^{b}$ and Rui Fausto*a \\ ${ }^{a}$ Department of Chemistry (CQC), University of Coimbra, 3004-535 Coimbra, Portugal. \\ E-mail:rfausto@ci.uc.pt \\ ${ }^{b}$ Department of Chemistry, University of Sofia, 1164 Sofia, Bulgaria
}

Received 29th May 2001, Accepted 31st July 2001

First published as an Advance Article on the web 7th September 2001

\begin{abstract}
Study of the conformational isomerism of methyl cyanoacetate $\left(\mathrm{N} \equiv \mathrm{CCH}_{2} \mathrm{COOCH}_{3}, \mathrm{MCA}\right)$ aided for the first time by matrix-isolation infrared spectroscopy is reported. The conformational isomerization processes in MCA in the temperature range from 10 to $70 \mathrm{~K}$ were studied in detail in argon and xenon matrixes. During annealing of the matrixes direct interconversion of the gauche into the syn conformer has been registered. A similar, but more pronounced, gauche $\rightarrow$ syn interconversion effect was observed in a series of experiments in which matrixes were deposited at increased substrate temperature. The experiment is supported by theoretical predictions undertaken at different levels of approximation (MP2 and DFT/B3LYP). It is shown that, for the single molecule in vacuum, the syn conformer $\left(\mathrm{C}-\mathrm{C}-\mathrm{C}=\mathrm{O}\right.$ dihedral angle equal to $\left.0^{\circ}\right)$ corresponds to the conformational ground state, with the doubly degenerated by symmetry gauche conformer $(\mathrm{C}-\mathrm{C}-\mathrm{C}=\mathrm{O}$ dihedral angle equal to $\pm 139.9^{\circ}$ ) being only slightly less stable than the most stable form (MP2/6-31G** $\left.\Delta E_{(\text {gauche } \rightarrow \text { syn })}=0.174 \mathrm{~kJ} \mathrm{~mol}^{-1}\right)$. In the matrixes, the energy gap between the syn and gauche conformers increases, with $\Delta E_{(\text {gauche } \rightarrow \text { syn })}$ estimated to be about $1.4 \mathrm{~kJ} \mathrm{~mol}^{-1}$ in xenon. The predicted energy barriers for conformer interconversion were found to be significantly low: the MP2/6-31G** calculated $\Delta E_{(\text {syn } \rightarrow \text { gauche })}$ energy barrier is $c a .3 .65 \mathrm{~kJ} \mathrm{~mol}^{-1}$, while the calculated energy barrier separating the two symmetrically equivalent gauche conformers stays only $0.196 \mathrm{~kJ} \mathrm{~mol}^{-1}$ above the energy of these forms. In the matrixes, $\Delta E_{(\text {syn } \rightarrow \text { gauche })}$ energy barrier increases to at least $17-18 \mathrm{~kJ} \mathrm{~mol}^{-1}$, while the $\Delta E_{\text {(gauche } \rightarrow \text { gauche })}$ energy barrier is, with all probability, still lower than in the gaseous phase. The matrix isolation spectroscopic data indicate that interconversion between the gauche conformers occurs even in the low temperature matrixes.
\end{abstract}

\section{Introduction}

Methyl cyanoacetate $\left(\mathrm{N} \equiv \mathrm{CCH}_{2} \mathrm{COOCH}_{3} ; \mathrm{MCA}\right)$ is of considerable interest as a reagent and intermediate product in pharmaceutical organic synthesis as well as in the synthesis of some biologically active compounds used in agriculture. ${ }^{1}$ Regarding the conformational isomerism, this compound was first studied by Charles et al., ${ }^{2}$ and Sinha and Katon ${ }^{3}$ using vibrational spectroscopy in the condensed phases. Later on, Leibowitz et al. ${ }^{4}$ undertook a detailed infrared study on the conformational isomerism in MCA dissolved in liquid argon and reviewed the previously reported vibrational data for the condensed phases using both Raman and infrared spectroscopy, supported by ab initio MO calculations undertaken at the HF/4-21G level of theory. In addition, they have also obtained the infrared spectrum of the vapour phase. ${ }^{4}$ More recently, this compound was also studied in our laboratory, in the liquid and solid states, using infrared and Raman spectroscopies and quantum chemical calculations performed at the $\mathrm{HF} / 6-31 \mathrm{G}^{*}$ level of approximation. ${ }^{5}$

From the previously reported studies, the following conclusions were drawn:

(a) In the liquid phase, as well as in solution and in the amorphous solid, ${ }^{2-4}$ MCA was shown to exist as a mixture of two conformational states of similar energies, differing by the relative orientation of the $\mathrm{NC}-\mathrm{C}-\mathrm{C}=\mathrm{O}$ axis (Fig. 1). In the neat liquid, the enthalpy differences obtained experimentally

$\dagger$ Electronic Supplementary Information available. See http:// www.rsc.org/suppdata/cp/b1/b104671n/ by several authors range from $2.0^{5}$ to $4.06 \mathrm{~kJ} \mathrm{~mol}^{-1}{ }^{2}$ with the syn conformer being the most stable form and the doubly degenerated by symmetry gauche form corresponding to the second observed conformational state.

(b) In the crystalline phase, only the syn conformer exists, ${ }^{5}$ as is indicated by the absence of the bands ascribable to the gauche conformer in the spectrum of this phase.

(c) In the gaseous phase, both syn and gauche conformers were observed experimentally. ${ }^{4}$ On the basis of asymmetric top contour simulations of the band assigned to the $\mathrm{CH}_{3}$ symmetric stretching vibration in the spectrum of MCA in the gas phase and on the absolute intensities of the bands assigned to the $v \mathrm{C} 1-\mathrm{C} 5$ vibration of each conformer $\left(\mathrm{syn}: 893 \mathrm{~cm}^{-1}\right.$; gauche: $854 \mathrm{~cm}^{-14}$ ), it was proposed by Leibowitz et al. ${ }^{4}$ that, in this phase, the gauche form should be the most stable of the two conformers observed. However, the theoretical predictions for the relative stability of the two observed conformers contradict the above conclusion, predicting the syn form as the conformational ground state for the isolated molecule situation $\left(\Delta E_{\text {gauche-syn }}: 0.94 \mathrm{~kJ} \mathrm{~mol}^{-1} \mathrm{HF} / 6-31 \mathrm{G}^{*} ; 53.92 \mathrm{~kJ}\right.$ $\left.\mathrm{mol}^{-1} \mathrm{HF} / 4-21 \mathrm{G}^{4}\right)$.

(d) The syn conformer is stabilised in more polar media relative to the gauche form, since it has a considerably larger dipole moment $\left(5.71 \mathrm{D}^{5}\right)$ than the latter form $\left(2.95 \mathrm{D}^{5}\right)$.

(e) Besides internal rotation around the $\mathrm{C} 1-\mathrm{C} 5$ bond, in $\mathrm{MCA}$, internal rotation around the $\mathrm{C} 1-\mathrm{O} 3$ bond also leads to conformational isomerism. ${ }^{5}$ However, the possible conformers of MCA exhibiting an s-trans $\mathrm{O}=\mathrm{C}-\mathrm{O}-\mathrm{C}$ axis $(\mathrm{O}=\mathrm{C}-\mathrm{O}-\mathrm{C}$ dihedral angle equal to $180^{\circ}$ ) were found to be of no practical interest, because their energies are higher by more than $35 \mathrm{~kJ}$ 


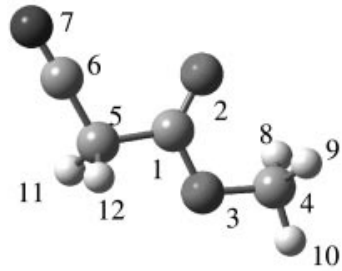

syn
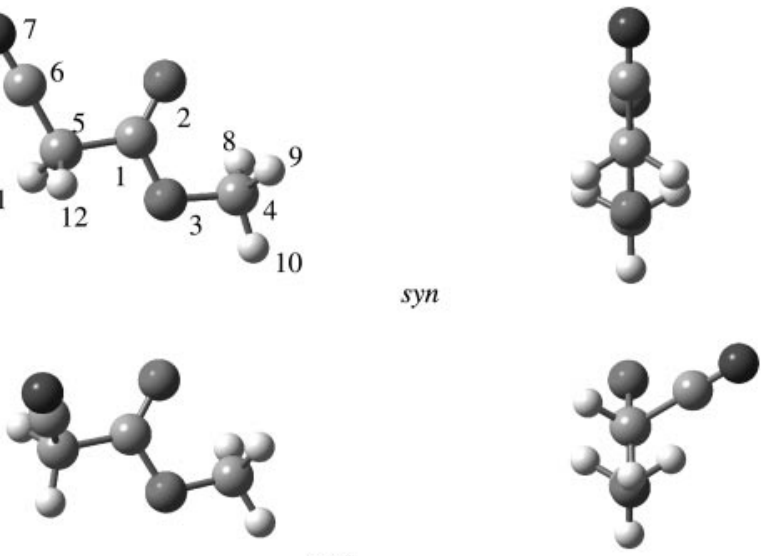

$\mathrm{TS}_{\text {syn } \rightarrow \text { gauche }}$
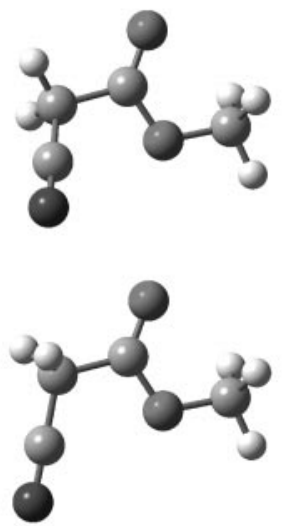

gauche
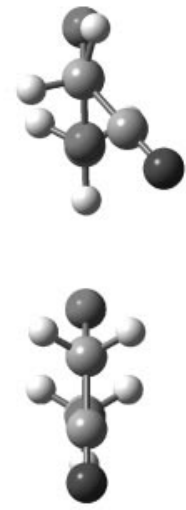

$\mathrm{TS}_{\text {gauche } \rightarrow \text { gauche }}$

Fig. 1 Relevant conformations of methyl cyanoacetate and atom numbering scheme.

$\mathrm{mol}^{-1}$ than the energy of the $s$-cis conformers. ${ }^{5}$ The preferred orientation of the methyl ester group in MCA was found to be that usually observed in simple methyl esters, with one of the hydrogen atoms placed in the anti periplanar position relative to the carbonyl carbon atom. ${ }^{6-8}$

(f) To the best of our knowledge no published experimental data on resolved vibrational spectra for isolated MCA molecules are available.

In the present work, the conformational isomerism in MCA is studied in order to obtain a more consistent picture, in particular with regard to what concerns the relative stability of the observed conformers. With this in perspective, the method of matrix isolation infrared spectroscopy was used in this study together with new higher-level theoretical calculations. In order to estimate both conformer energy differences and barriers of interconversion, vibrational spectra of MCA isolated in low temperature $\mathrm{Ar}$ and Xe matrixes under different deposition conditions were recorded for the first time. Annealing of the matrixes was also carried out systematically. The theoretical data were obtained using both the second-order Møller-Plesset perturbation method (MP2) and Becke-style 3parameter density functional theory, with the Lee-Yang-Parr correlation functional (B3LYP). As will be shown below, the present study unequivocally proves that the syn conformer is the lowest energy conformational state in MCA and that interconversion between the gauche conformers occurs even in the low temperature matrixes.

\section{Experimental and computational methods}

Methyl cyanoacetate (Aldrich, spectroscopic grade) was preliminary purified by vacuum distillation. In order to remove any dissolved gaseous impurities the compound was also sub- jected to multiple freeze-pump-thaw cycles before cooling the cryostat and starting the matrix deposition procedure. A glass vacuum system and standard manometric procedures were used to deposit matrix gases (argon, Air Liquid, 99.9999\%; xenon, Air Liquid, 99.995\%), which were used without further purification. Gas deposition rate during sample preparation was $c a .10 \mathrm{mmol} \mathrm{h}^{-1}$. MCA was codeposited from a specially designed Knudsen cell with shut-off possibility, the principal component of which is a SS-4BMRG micrometer valve (NUPRO). Further details of the experimental setup can be found elsewhere. ${ }^{9}$

The infrared spectra in the range $4000-400 \mathrm{~cm}^{-1}$ were obtained using a Mattson (Infinity 60AR Series) Fouriertransform infrared spectrometer equipped with a deuterated triglycine sulphide (DTGS) detector and $\mathrm{Ge} / \mathrm{KBr}$ beamsplitter. Data collection was performed with $0.5 \mathrm{~cm}^{-1}$ spectral resolution. All experiments were done on the basis of an APD Cryogenics close-cycle helium refrigeration system with a DE-202A expander. Necessary modifications of the sample compartment of the spectrometer were made in order to accommodate the cryostat head and allow efficient purging of the instrument by a stream of dry air to remove water and $\mathrm{CO}_{2}$ vapours.

Experimental intensities were determined from the area below the observed bands which, whenever necessary, were subjected to previous deconvolution by using the PEAKFIT module of the Grams/32 AI (version 6.0) program. ${ }^{10}$

Theoretical calculations were performed with the GAUSSIAN98 program package ${ }^{11}$ at two levels of theory: Becke-style 3-parameter density functional theory using the Lee-Yang-Parr correlation functional (B3LYP) ${ }^{12-14}$ and second order Møller-Plesset perturbation theory (MP2) using the $6-31 \mathrm{G}^{*}$ and $6-31 \mathrm{G}^{* *}$ basis sets. ${ }^{15,16}$ Molecular geometries were fully optimised by the force gradient method using Berny's algorithm ${ }^{17}$ using the tight built in convergence criteria for the geometry optimisation, both at the B3LYP and MP2 levels of theory. The precise nature of the optimised stationary points was determined by analysis of the corresponding Hessian matrices.

The calculated (B3LYP/6-31G*) wavenumbers were fitted to experimental data yielding $v_{\text {fitted }}=0.9481 v_{\mathrm{B} 3 \mathrm{LYP}}+27.1$, with $R^{2}=0.999845$. The gravity centres of the observed features $\left(v_{\mathrm{gc}}=\Sigma\left(I_{i} v_{i}\right) / \Sigma I_{i}\right.$, where the sum extends to all component bands assigned to a given vibration and $I_{i}$ stands for the integrated intensity of the component $i$ ), extracted from the spectrum obtained in argon using a deposition temperature of $10 \mathrm{~K}$ and the valve nozzle at $c a .293 \mathrm{~K}$, were used in the fitting procedure. The mean relative error in the fitted wavenumbers is less than $0.5 \%$.

\section{Results and discussion}

Table 1 summarizes the energetic results of the theoretical calculations carried out at different levels of approximation. Corresponding geometries can be found in Table S2 (see the Electronic Supplementary Information (ESI) $\dagger$ ). Fig. 2 presents the MP2/6-31G** calculated potential energy profile for internal rotation around the $\mathrm{C} 1-\mathrm{C} 5$ bond. In consonance with the previously published results, ${ }^{4,5}$ both the B3LYP and MP2 calculations predict the existence of two distinct stable conformations differing by internal rotation around the $\mathrm{C} 1-\mathrm{C} 5$ bond (see Fig. 1): syn $\left(\angle \mathrm{C}-\mathrm{C}-\mathrm{C}=\mathrm{O}, 0^{\circ}\right)$ and gauche $(\angle \mathrm{C}-\mathrm{C}-\mathrm{C}=\mathrm{O}$, ca. $\left.\pm 140^{\circ}-\mathrm{MP} 2\right)$. When energies are corrected by zero-point vibrational energy contributions, both B3LYP and MP2 calculations predict the syn form as being the conformational ground state. However, in all cases the estimated conformational energy differences are very small $\left(<0.2 \mathrm{~kJ} \mathrm{~mol}^{-1}\right)$ and no conclusive answer regarding the relative stability of the different conformers can be extracted by taking into consideration only the theoretical results. 
Table 1 Calculated relative energies $\left(\mathrm{kJ} \mathrm{mol}^{-1}\right)$ for the relevant conformations of methyl cyanoacetate

\begin{tabular}{|c|c|c|c|c|}
\hline & syn & $\mathrm{TS}_{(\text {syn } \rightarrow \text { gauche })}$ & gauche & $\mathrm{TS}_{(\text {gauche } \rightarrow \text { gauche })}$ \\
\hline$\Delta E_{\text {electron }}\left(\mathrm{B} 3 \mathrm{LYP} / 6-31 \mathrm{G}^{*}\right)$ & 0.000 & & -0.717 & \\
\hline$\Delta$ ZPVE (B3LYP/6-31G*) & $0.000^{a}$ & & $0.857^{b}$ & \\
\hline$\Delta E+\Delta \mathrm{ZPVE}(\mathrm{B} 3 \mathrm{LYP})$ & 0.000 & & 0.140 & \\
\hline$\Delta E_{\text {electron }}\left(\mathrm{MP} 2 / 6-31 \mathrm{G}^{* *}\right)$ & 0.000 & 3.022 & -0.553 & 0.357 \\
\hline$\Delta \mathrm{ZPVE}^{\prime c}\left(\mathrm{MP} 2 / 6-31 \mathrm{G}^{* *}\right)$ & 0.000 & 0.762 & 0.662 & 0.153 \\
\hline$\Delta \mathrm{ZPVE}\left(\mathrm{MP} 2 / 6-31 \mathrm{G}^{* *}\right)$ & $0.000^{d}$ & & $0.727^{e}$ & \\
\hline$\Delta E_{\text {electron }}+\Delta \mathrm{ZPVE}(\mathrm{MP} 2)$ & 0.000 & & 0.174 & \\
\hline
\end{tabular}

${ }^{a}$ The contribution associated with the $\mathrm{C}-\mathrm{C}$ torsion $\left(h v_{\mathrm{C}-\mathrm{C}} / 2\right)$ is $0.191 \mathrm{~kJ} \mathrm{~mol}^{-1} \cdot{ }^{b}$ The contribution associated with the $\mathrm{C}-\mathrm{C}$ torsion $\left(h v_{\mathrm{C}-\mathrm{C}} / 2\right)$ is $0.126 \mathrm{~kJ} \mathrm{~mol}^{-1} \cdot{ }^{c} \mathrm{ZPVE}^{\prime}$ corresponds to the total zero-point vibrational energy minus the contribution associated with the $\mathrm{C}-\mathrm{C}$ torsion $\left(h v_{\mathrm{C}-\mathrm{C}} / 2\right)$.

${ }^{d}$ The contribution associated with the $\mathrm{C}-\mathrm{C}$ torsion $\left(h v_{\mathrm{C}-\mathrm{C}} / 2\right)$ is $0.140 \mathrm{~mol}^{-1}$. ${ }^{e}$ The contribution associated with the $\mathrm{C}-\mathrm{C}$ torsion $\left(h v_{\mathrm{C}-\mathrm{C}} / 2\right)$ is 0.205 $\mathrm{kJ} \mathrm{mol}^{-1}$.

A detailed analysis of the molecular geometries of the conformers of MCA was made in our previous work $\left(\mathrm{HF} / 6-31 \mathrm{G}^{*}\right.$ calculated data ${ }^{5}$ ) and will not be repeated here.

A typical matrix isolation FTIR spectrum of MCA trapped in an Ar matrix is shown in Fig. 3A. Tables S3 and S4 (ESI $\dagger$ ) present the vibrational data obtained in this study. Besides the experimental data obtained in $\mathrm{Ar}$ and $\mathrm{Xe}$ matrixes, these

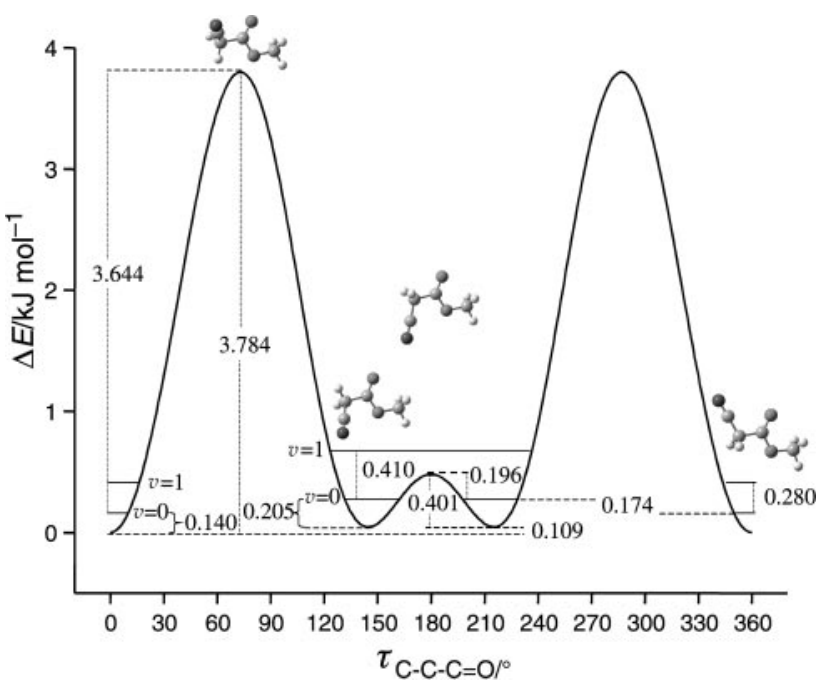

Fig. 2 MP2/6-31G** potential energy profile for internal rotation around $\mathrm{C} 1-\mathrm{C} 5$.

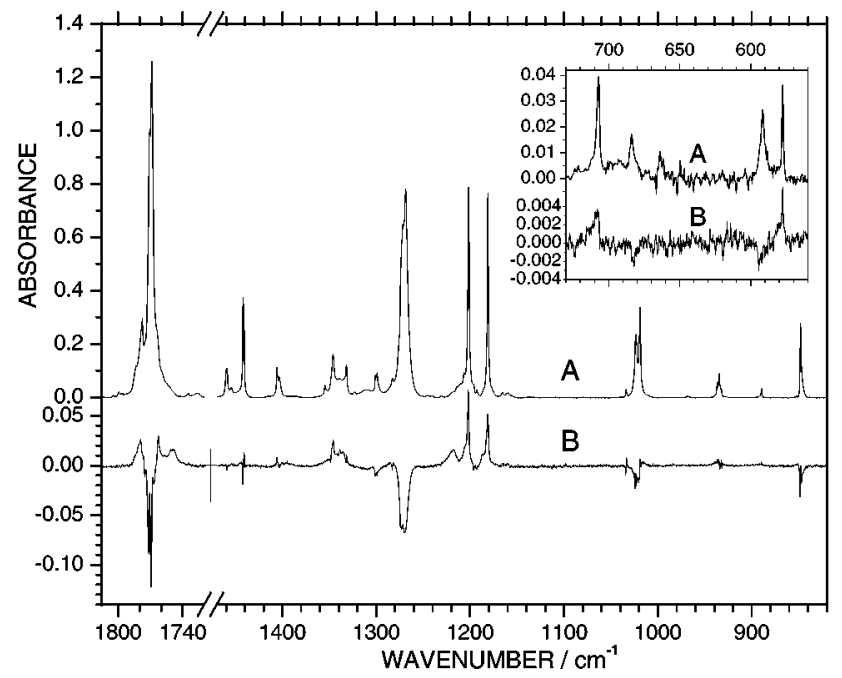

Fig. 3 A, FTIR spectrum (fingerprint region) of methyl cyanoacetate in argon matrix. Knudsen cell nozzle kept at $293 \mathrm{~K}$ and cold substrate at $10 \mathrm{~K}$ during deposition. B, Difference spectrum between the same sample annealed to $18 \mathrm{~K}$ and A. Positive peaks correspond to the absorptions which increase after annealing. The weak band at 663.8 $\mathrm{cm}^{-1}$ corresponds to absorption of matrix-isolated $\mathrm{CO}_{2}$. tables also contain the results of the theoretical calculations and the proposed assignments.

In a first series of experiments, different matrixes of MCA in argon were prepared by varying the temperature of the nozzle from $c a .293 \mathrm{~K}$ to $c a .373 \mathrm{~K}$. The resulting spectra, obtained using the same deposition conditions $(10 \mathrm{~K})$ and matrix gas, do not show any visible difference. This is in consonance with the predicted small energy difference between the two conformers in the gas phase. Indeed, the predicted change in the relative population ratio, $P_{\text {gauche }}: P_{s y n}$, upon increasing the temperature from 293 to $373 \mathrm{~K}$, assuming Boltzmann distribution and taking into consideration the different degeneracy of the conformational levels $\left(g_{s y n}=1 ; g_{\text {gauche }}=2\right)$ and the calculated energy difference $\left(\Delta E_{\text {gauche-syn }}=0.174 \mathrm{~kJ} \mathrm{~mol}^{-1}\right)$, is less than $3 \%$, and then below the experimental limits of detection.

According to the calculations, the relative population $P_{\text {gauche }}: P_{\text {syn }}$, at $293 \mathrm{~K}$ is 1.86 . Using this value as weight factor, the ratio between the predicted sum of intensities of all bands which could be assigned to individual conformers in the observed spectra $\left(v \mathrm{CN}, v \mathrm{C}=\mathrm{O}, \delta \mathrm{CH}_{3}\right.$ as $\mathrm{A}^{\prime}, \delta \mathrm{CH}_{2}, \omega \mathrm{CH}_{2}$, $\nu \mathrm{C} 1-\mathrm{O} 3, \gamma \mathrm{CH}_{3} \mathrm{~A}^{\prime}, \gamma \mathrm{CH}_{2}, \nu \mathrm{C} 1-\mathrm{C} 5, \nu \mathrm{OCO}$ and $\gamma \mathrm{C}=\mathrm{O}$; see Tables S3 and $\mathrm{S} 4 \dagger), I_{\text {gauche }}: I_{\text {syn }}$, was calculated. This value was then compared with the measured total intensity ratio in the spectra of freshly prepared matrixes of MCA in argon and xenon (the temperatures of deposition were, respectively, 10 and $20 \mathrm{~K}$ ). The value obtained for the argon matrix is, within experimental error, equal to the calculated value, indicating that in this case the gas phase equilibrium could be efficiently trapped in the matrix. The same situation has been observed for other carboxylic acid monomers (e.g., oxalic, malonic and acrylic acids ${ }^{18-20}$ ) when similar deposition conditions were used. These results clearly indicate that the theoretically predicted relative energy of the two observed conformers is a good estimation of the corresponding value in the gaseous phase. On the other hand, the measured intensity ratio in the spectra obtained in xenon was found to be $c a .15 \%$ smaller than in argon, indicating that, in this case, the gauche conformer was partially converted into the syn form during deposition. We shall return to this point later.

The spectrum presented in Fig. 3A of MCA isolated in argon corresponds to a nozzle temperature of $c a .293 \mathrm{~K}$ and substrate temperature of $10 \mathrm{~K}$. After completing deposition, the matrix was submitted to a step-by-step annealing procedure, using increments of $2-3 \mathrm{~K}$. At a temperature of $c a$. 15 $\mathrm{K}$, a discernible rearrangement of the relative intensities of the bands could be observed, the bands ascribable to the syn conformer increasing their intensity and those due to the second conformer decreasing. Upon annealing to higher temperatures the intensity ratios of the bands due to the conformers continue to change systematically favouring the syn form (see Fig. 3B), while absorptions due to aggregation start to increase at a temperature of $c a .18 \mathrm{~K}$ (e.g., bands in the 1763-1748, 1360 $1330,1220-1206 \mathrm{~cm}^{-1}$ regions and the pair of bands at 1187 and $1185 \mathrm{~cm}^{-1}$ are band marks due to aggregated species, as 
confirmed by annealing of the matrixes to the highest available temperatures).

The observed changes in the relative band intensities clearly indicated that the relative populations of the conformers initially present in the matrix had been changed, with the less stable conformer (gauche) being converted into the most stable form (syn). Hence, these experiments confirm the theoretical predictions, clearly showing that the syn form corresponds to the most stable conformer of MCA monomeric species.

Annealing experiments were also carried out for MCA in xenon. The spectra obtained in xenon are essentially the same as in argon although the usual red shifts in the band maxima due to the higher polarizability of the Xe atom when compared with $\mathrm{Ar}^{19}$ can be easily noticed. The discussion below will be focused mainly on analysis of the data obtained in Xe, where the range of temperatures available both to deposit the sample and to undertake annealing experiments is considerably wider.

As was already mentioned, the ratio of the conformers trapped in the xenon matrix, when the temperature of the substrate was $20 \mathrm{~K}$, was found to be shifted in favour of the syn isomer with respect to the gas phase equilibrium at the temperature of the nozzle. This observation indicates that partial conversion from the gauche to the syn form must have occurred during deposition. This can be clearly shown, for instance, by comparing the carbonyl stretching region in the spectra of the non-annealed matrixes of MCA in argon (Fig. $3 \mathrm{~A})$ and xenon (Fig. 4, trace 1). The relative intensities of the bands appearing at higher frequencies (pairs of bands at 1783/ $1777 \mathrm{~cm}^{-1}$ in argon and $1780 / 1770 \mathrm{~cm}^{-1}$ in xenon), that are ascribed to the syn conformer, are clearly less intense in the argon than in the xenon spectrum, when compared with the intensities of the lower frequency $v \mathrm{C}=\mathrm{O}$ bands due to the gauche conformer (Ar: 1770/1768 $\mathrm{cm}^{-1}$; Xe: 1762/1751 $\left.\mathrm{cm}^{-1}\right)$. Indeed, the ratios of the total integrated intensities are $I_{\text {syn }} / I_{\text {gauche }}(\mathrm{Ar})=0.999 / 4.860=0.21$ and $I_{\text {syn }} / I_{\text {gauche }}(\mathrm{Xe})=$ $1.269 / 2.454=0.52$ (see Tables S3 and S4 $\dagger$ ). In xenon, partial conversion of the gauche conformer into the syn form was observed already at $20 \mathrm{~K}$, while absorptions due to aggregation start to increase at a temperature of $c a .35 \mathrm{~K}$.

The $v \mathrm{C}=\mathrm{O}$ stretching region (Fig. 4 and Fig. $\mathrm{S} 5 \mathrm{~B} \dagger$ ) is very illustrative and exhibits the three main patterns of behaviour of spectral bands during matrix annealing. First, the intensities of the bands ascribed to the most stable syn form (1780/ $1770 \mathrm{~cm}^{-1}$ ) increase with increasing substrate temperature up to $40 \mathrm{~K}$ (see traces 1-3 in Fig. 4). In this temperature range the dominating process is the conversion of the gauche form into

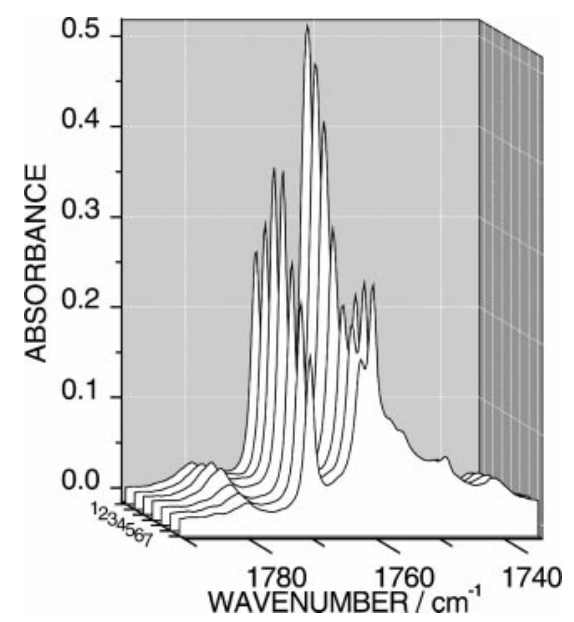

Fig. 4 FTIR spectrum of the carbonyl stretching vibration of methyl cyanoacetate isolated in xenon matrix. "Annealing series". Trace 1, spectrum recorded immediately after deposition (cold substrate kept at $20 \mathrm{~K}$ during deposition). Traces $2-7$, spectra of the same sample annealed to $30,40,50,60,65$ and $70 \mathrm{~K}$, respectively. the syn isomer. At substrate temperatures higher than $40 \mathrm{~K}$ the monomeric syn form is consumed by association processes and the intensity of the corresponding bands $(1780 / 1770$ $\mathrm{cm}^{-1}$ ) decreases (traces 4-7 in Fig. 4). Second, the bands due to the gauche conformer (e.g., $1762 \mathrm{~cm}^{-1}$ ) decrease continuously in intensity with increase of the substrate temperature. This decrease is due to both gauche $\rightarrow$ syn interconversion and association. Third, the bands due to aggregated species (e.g., at $1760,1748,1740 \mathrm{~cm}^{-1}$ ), start to increase significantly in intensity at a temperature of $c a .35 \mathrm{~K}$, since aggregates are produced from both syn and gauche conformers. These three types of behaviour are characteristic and were observed throughout the entire spectrum. Based on these observations we were able to distinguish unequivocally between the absorption bands of the syn and gauche conformers.

The possibility of observing conformational isomerization processes by annealing the matrixes depends on the energy barriers for the internal rotation and, as pointed out by Barnes, ${ }^{21}$ a rough estimation of these energy barriers could be made by determining the temperature at which the processes start to occur. True equilibrium in the matrix would require that the barriers for both forward and reverse conformational isomerization processes could be overcome at a given temperature. Then, above the minimum temperature satisfying this condition the changes in the relative populations of the conformers, due to annealing at different temperatures, must be reversible. Such phenomena were observed, for instance, for some simple alcohol molecules, like methanol or ethanol, isolated in different matrixes. ${ }^{2,23}$ In all those cases where true equilibrium has been observed in low temperature matrixes, the gas-phase energy barriers are known to be relatively low (below $6 \mathrm{~kJ} \mathrm{~mol}^{-1}$ ) and, most times, only hydrogen atoms are involved in the movement leading to the conformational changes, i.e., no significant matrix relaxation is required during the conformational isomerization process.

Indeed, it is well known ${ }^{24-27}$ that the matrix may affect, to some extent, the relative energies of the conformers and the energy barriers for isomerization, when compared with the values relative to the individual molecule in the gaseous phase (or in vacuum). Usually, more polar forms are stabilized in the matrixes (with respect to the gas phase), and this stabilization increases with the polarizability of the matrix ${ }^{18,28}$ (e.g., the effect can be expected to be more pronounced in Xe than in Ar matrixes). In turn, the rigidity of the matrix usually leads to higher energy barriers than observed in the gas phase. Moreover, since decreasing the temperature of the matrix makes it more rigid, it could also be expected that the energy barriers would increase upon lowering the temperature of the matrix. Another important point that must also be taken into account is the fact that, in a matrix, the energy of the guest molecules may be affected by different possible packing arrangements of the host molecules around them, as a result of the rapid freezing. ${ }^{24-27}$ In other words, the molecules of the solute assume a distribution of energies resulting from the interactions with a variety of possible matrix surroundings.

In the case of MCA, there are three different possible isomerization processes. The first corresponds to the above discussed gauche $\rightarrow$ syn conversion, the second, to the inverse reaction, syn $\rightarrow$ gauche and the third, to the gauche $\rightarrow$ gauche interconversion. The predicted gas-phase energy barrier associated with this latter process is extremely small $(0.196 \mathrm{~kJ}$ $\mathrm{mol}^{-1}$ ) being below the first excited $\tau \mathrm{C} 1-\mathrm{C} 5$ torsional level (see Table 1 and Fig. 2). Even though we cannot rule out the possibility of this energy barrier being slightly higher in the matrixes, a change in the opposite direction appears to be more probable. Indeed, the transition state for this process corresponds to the symmetric anti conformation (Fig. 1), where all heavy atoms stay in the same plane, and this conformation shall perturb the matrix packing less, as shown previously for other molecules, in particular for derivatives of 
DNA bases exhibiting planar and non-planar heavy atoms backbones ${ }^{29}$ and for 1,3-butadiene. ${ }^{30}$ As will be pointed out in detail below, the experimental data clearly indicate that free interconversion between the two equivalent-by-symmetry gauche forms occurs in the low temperature matrixes, resulting in the fact that the observed spectroscopic properties of the second conformational state resemble more those predicted for the anti structure than those calculated for the minimum energy gauche conformation.

In relation to the relative heights of the barriers for interconversion between the gauche and syn forms in the gas phase and in the low temperature matrixes, a reasoning similar to that presented above for the gauche $\rightarrow$ gauche interconversion can be made. In this case, it can be expected that the energy barriers are larger in the matrixes, since the transition state is an asymmetric structure. This effect will be more pronounced for the syn $\rightarrow$ gauche conversion than for the inverse reaction, since the syn conformer has a planar heavy atom backbone, favouring better matrix packing. In addition, this conformer is the most polar form (the MP2/6-31G** calculated dipole moments for syn and gauche conformers are 5.14 and $2.88 \mathrm{D}$ ) and, as discussed above, will be stabilized in the matrixes with respect to the gauche form, as well as to the syn $\rightarrow$ gauche transition state, whose calculated dipole moment is $3.40 \mathrm{D}$.

If the Barnes relationship ${ }^{21}$ is used to estimate the gauche$\rightarrow$ syn energy barrier from the temperature at which this isomerization process starts to be observed in the matrixes $(15-20 \mathrm{~K})$ a value of $c a .5 \mathrm{~kJ} \mathrm{~mol}^{-1}$ is obtained. However, we shall consider this value as a lower limit for the effective barrier in the matrixes, since we can expect, as discussed previously, that the first molecules which are able to surpass the energy barrier for isomerization are those that were trapped in less favourable conditions (i.e., the fraction of molecules that is more distant from a true equilibrium situation relative to the surroundings and then has a higher energy). We shall notate this value as $\Delta E_{\text {gauche }}^{\neq} \rightarrow$ syn , where the symbol * stands for the "hottest" molecule in the ensemble constituted by the whole set of trapped guest molecules. The corresponding lower limit value for the energy barrier for the reverse process $\left(\Delta E_{s y n^{*} \rightarrow \text { gauche }}^{\neq}\right)$can now be estimated to be ca. 6-7 $\mathrm{kJ} \mathrm{mol}^{-1}$, since in the neat liquid the energy difference between the gauche and the syn conformers increases by $c a .1 .8 \mathrm{~kJ} \mathrm{~mol}^{-1}$ relative to the predicted value for the gas-phase $\left(\Delta E_{g a c h e-s y n}\right.$ in the liquid phase was found to be $\left.2.0 \pm 0.2 \mathrm{~kJ} \mathrm{~mol}^{-1}\right),{ }^{5}$ and with all probability the relative stabilization of the syn form with respect to the gauche conformer in the matrixes shall be smaller than in the liquid phase.

In order to test if true equilibrium between the syn and the gauche forms was achieved in the matrixes, an annealing and freezing cycle was undertaken, where, after increasing the temperature of the matrix from the deposition temperature to the maximum temperature at which no significant increase of the bands due to aggregates are observed, the temperature was subsequently reduced to the initial value. The time spent at each temperature was kept constant and equal to $c a .30 \mathrm{~min}$. In these experiments, while the gauche $\rightarrow$ syn isomerization could be clearly observed upon increasing the temperature, no changes could be noticed during the further cooling steps.

To interpret the fact that the observed gauche $\rightarrow$ syn isomerization is not reversible under the experimental conditions used we should first remember that, as discussed above, in the matrixes the trapped molecules assume a distribution of energies resulting from their interactions with the matrix. At $25 \mathrm{~K}$, an energy barrier of $c a .8 \mathrm{~kJ} \mathrm{~mol}^{-1}$ is able to prevent isomerization. ${ }^{21}$ Thus, if the true gauche $\rightarrow$ syn energy barrier in the matrixes (measured taken as reference those molecules that were trapped more favourably) is larger than this value, only part of the reactant molecules can convert to the syn conformer. Since we started with a relative conformational population that is close to that of the gas phase equilibrium, this is equivalent to saying that at each point, during the heating cycle, the population of the gauche form is still in excess relative to that of the most stable syn form. So, any subsequent decrease in temperature cannot lead to observation of any further conformational isomerization.

Since the isomerization reactions are first order, an exponential decay of the gauche conformer towards the syn form is expected and thus, in order to check the above hypothesis, an indirect experimental method was chosen that avoids the need to use impractical long time steps during the annealing procedure. Hence, different substrate temperatures during deposition were used to prepare the matrixes, with all other relevant variables kept constant. The spectra of the matrixes obtained by deposition at substrate temperatures $20,30,40$ and $50 \mathrm{~K}$ are presented in Fig. 5 and Fig. S5A-E $\uparrow$. The spectra were normalized to the same peak intensity as that of the band of the syn conformer at $1179.8 \mathrm{~cm}^{-1}$. This band was chosen because it does not overlap with absorptions due to either the gauche conformer or to associates. In this way of representation the changes in the relative populations of the two conformers from one matrix deposition to the other are clearly seen. With variation of the substrate temperature the bands at 1350-1330 and $1210-1190 \mathrm{~cm}^{-1}$ do not change their relative intensity in comparison to the $1179.8 \mathrm{~cm}^{-1}$ band. Thus these three band sets belong to the same species. During the annealing experiments described above these bands follow the behavior pattern of the syn form. The band centered at 1262.3 $\mathrm{cm}^{-1}$, which appears very strong in the matrix deposited at 20 $\mathrm{K}$, decreases drastically in intensity with increase in substrate temperature, vanishing almost completely in the spectrum of the matrix deposited at $50 \mathrm{~K}$. The less intense bands at 1304.7 , 1295.8 and $1287.4 \mathrm{~cm}^{-1}$ follow this behavior. Based on this observation as well as on the results of annealing experiments these bands were assigned to the gauche form. The broad band that appears at $1278 \mathrm{~cm}^{-1}$ at higher temperatures belongs to associates.

From these studies, the following observations were made: (i) upon increase in the temperature at which the matrix was deposited, the $P_{\text {gauche }}: P_{\text {syn }}$ population ratio becomes smaller; (ii) $P_{\text {gauche }}: P_{\text {syn }}$ resulting from deposition at a given temperature is considerably smaller than the population ratio attained by annealing the matrix (deposited with the substrate at $20 \mathrm{~K}$ ) to the same temperature (the observed decrease in $P_{\text {gauche }}: P_{\text {syn }}$ becomes more pronounced with temperaturesee Fig. 6); and (iii) annealing to higher temperatures (up to

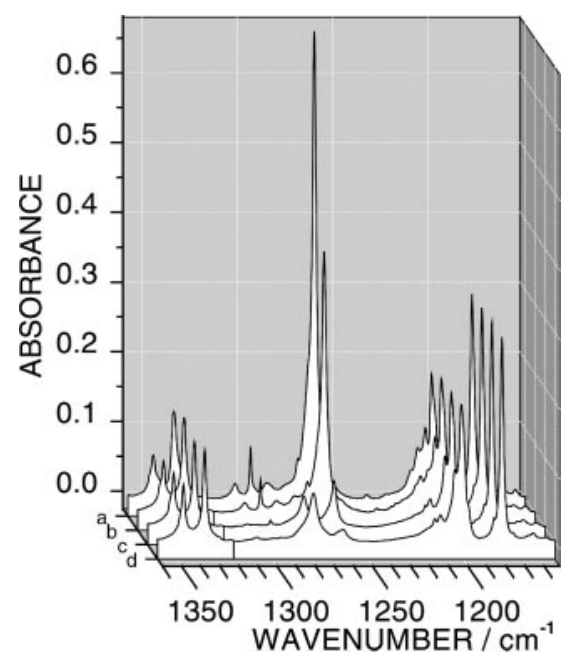

Fig. 5 FTIR spectra of methyl cyanoacetate in xenon matrix. "Deposition series". a, the same spectrum as Trace 1 in Fig. 4 (cold substrate kept at $20 \mathrm{~K}$ during deposition). b, c, d, cold substrate temperature during matrix deposition kept at 30,40 and $50 \mathrm{~K}$, respectively. All spectra normalized to the same peak intensity of the 1179.8 $\mathrm{cm}^{-1}$ absorption $\left(\mathrm{CH}_{3} \mathrm{~A}^{\prime}\right.$ rocking mode of $s y n-$ form) 


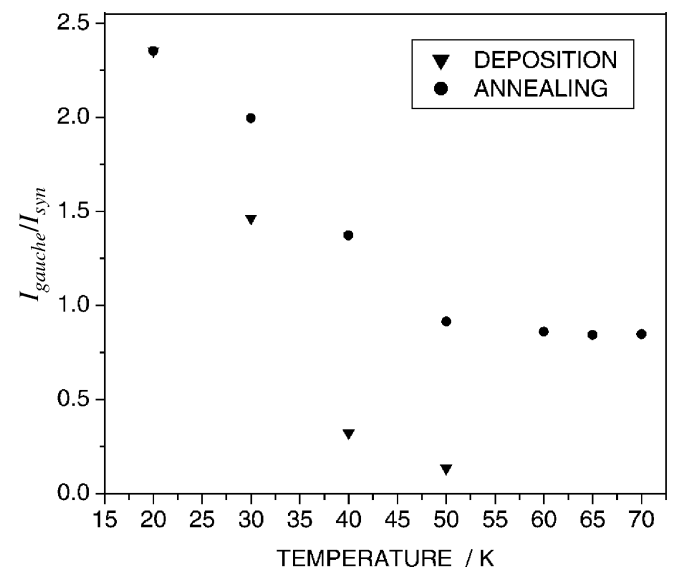

Fig. 6 Integral intensity ratio of the carbonyl stretching bands ascribed to gauche and syn forms of MCA in Xe matrix. ( $)$ Matrixes deposited at different substrate temperatures. (O) Annealing of the same matrix. Spectra were deconvoluted in order to account for overlapped bands.

ca. $70 \mathrm{~K}$ ) of the xenon matrix deposited at $50 \mathrm{~K}$ did not enable observation of the $s y n \rightarrow$ gauche isomerization - in fact, the dominant process at these highest temperatures is aggregation and gauche $\rightarrow$ syn isomerization is hardly observed.

As can easily be concluded, these observations fully support the above hypothesis. The lower $P_{\text {gauche }}: P_{\text {syn }}$ resulting from deposition with the substrate at a given temperature, when compared to the population ratio attained by annealing the matrix (deposited at $20 \mathrm{~K}$ ) to the same temperature, can be explained by considering that when deposition is made at higher temperatures the lower energy barriers associated with a less rigid matrix and the larger available energy at the arriving guest molecules both contribute to an increase in the number of molecules that can undergo isomerization towards the low temperature equilibrium. These results also clearly show that the $P_{\text {gauche }}: P_{\text {syn }}$, population ratio resulting from the annealing does not correspond to that of the equilibrium at each point during the heating cycle, and fully justifies the experimental observations under discussion.

As shown in (iii), at $70 \mathrm{~K}$ the true equilibrium was not yet attained. However, it is clear, taking into account the trends shown in Fig. 6, that the required temperature to attain this situation will not be much higher than this value. By the Barnes relationship, ${ }^{21}$ this means that the gauche $\rightarrow$ syn energy barrier in the studied matrixes (measured taking as reference those molecules that were trapped more favourably; i.e., from the bottom of the potential) is $c a .16 \mathrm{~kJ} \mathrm{~mol}^{-1}$. In addition, if we assume that the minimum $P_{\text {gauche }}: P_{s y n}$ value attained does not differ considerably from the true equilibrium population ratio at $50 \mathrm{~K}$, an estimation of the gauche-syn energy difference in xenon can be obtained. The resulting value $\left(c a .1 .4 \mathrm{~kJ} \mathrm{~mol}^{-1}\right)$ stays, as expected, between that calculated for the gas phase $\left(c a .0 .2 \mathrm{~kJ} \mathrm{~mol}^{-1}\right)$ and that obtained experimentally for the neat liquid $\left(2.0 \mathrm{~kJ} \mathrm{~mol}^{-1}\right){ }^{5}$ From the data above, the $s y n \rightarrow$ gauche energy barrier in xenon can also be estimated to be $c a .17-18 \mathrm{~kJ} \mathrm{~mol}^{-1}$.

It is important to stress that the experimental data clearly show that $\Delta E_{\text {gauche-syn }}$ must increase in the matrices when compared with the predicted value for the gas phase. In fact, if the energy difference between the two conformers was the same as predicted by the calculations for the gaseous phase, the equilibrium $P_{\text {gauche }}: P_{\text {syn }}$ population ratio should be larger than 1.3 , at $50 \mathrm{~K}$, i.e., the gauche form would be the most abundant conformer, which is clearly contradicted by the spectroscopic data obtained in the matrixes. Indeed, the experimental data show that at $50 \mathrm{~K}$ the population of the gauche conformer is lower than that of the syn form (see Fig. 4 and 5).
Comparing now $\Delta E_{\text {gauche } \rightarrow \text { syn }}^{\neq}$and $\Delta E_{s y n^{*} \rightarrow \text { gauche }}^{\neq}$with the corresponding values of the energy barriers from the bottom of the potential, it can be concluded that the difference in the interaction energies between the guest molecules and matrixes resulting from different local environment conditions amounts to $c a .11 \mathrm{~kJ} \mathrm{~mol}^{-1}$. This value compares well with available data on the relative energies of different relaxed matrix sites formed during trapping of a medium size organic molecule (a few tens of $\left.\mathrm{kJ} \mathrm{mol}^{-1}\right) .^{24}$

Once the details associated with the relative energies of the different relevant conformations of MCA in both the matrixes and in the gaseous phase are established, the complete assignment of its vibrational spectra (see Tables S3 and S4 $\dagger$ ) can be made with a higher degree of certainty.

The assignment of the bands due to the most stable conformer in the spectra of both xenon and argon matrixes could be undertaken easily, since these bands are intensified upon both annealing and increasing the deposition temperature. The bands decreasing in intensity are due to the second conformer. Most of the assignments now made confirm those previously made in ref. 4 and 5. However, some important changes were made concerning the assignment of the $\delta \mathrm{OC}=\mathrm{O}$ mode. This vibration is predicted to occur at $705.8 \mathrm{~cm}^{-1}$ in the syn conformer and at $709.2 \mathrm{~cm}^{-1}$ in the gauche structure (see Tables $\mathrm{S} 3$ and $\mathrm{S} 4 \dagger$ ), i.e., $\delta \mathrm{OC}=\mathrm{O}$ is predicted to occur at a slightly lower frequency in the syn than in the gauche form. However, the experimental data show the opposite trend: the band due to the syn form $\left(707.1 \mathrm{~cm}^{-1}\right.$, in argon) is observed at a higher frequency than that ascribable to the second conformer $\left(683.7 \mathrm{~cm}^{-1}\right.$, see inset in Fig. 3 and Figs. S5E and S4E $\dagger$ ). Very interestingly, the experimentally observed relative order of frequencies, both in $\mathrm{Ar}$ and in $\mathrm{Xe}$, is the same as that predicted for the anti conformation (calculated frequency: $677.5 \mathrm{~cm}^{-1}$ ), which corresponds to the transition between the two gauche forms. It is important to note that the predicted frequency of $\delta \mathrm{OC}=\mathrm{O}$ mode is expected to be most sensitive to the change of conformation from the gauche to the anti structure (together with the $\delta \mathrm{CCC}$ mode, which lies in a spectral region not accessible to our experiments-below $150 \mathrm{~cm}^{-1}$; see Table S4 $\dagger$ ). So, the experimental data clearly show that free interconversion between the two equivalent-by-symmetry gauche forms occurs in the low temperature matrixes, resulting in the observation of spectroscopic properties of the second conformational state which resemble more those predicted for the anti structure than those calculated for the minimum energy gauche conformation. These results are further supported by the fact that the bands ascribed to the less stable conformer, which are predicted to be more sensitive to the change in conformation, are those corresponding to the broadest observed bands (as could be expected, the band assigned to the $\delta \mathrm{OC}=\mathrm{O}$ mode corresponds the broadest observed band ascribed to a single vibration).

\section{Conclusion}

Study of the conformational isomerism of methyl cyanoacetate aided for the first time by matrix-isolation infrared spectroscopy is reported. The conformational isomerization processes in MCA in the temperature range 10 to $70 \mathrm{~K}$ were studied in detail in both argon and xenon matrixes. Direct interconversion of the gauche into the syn conformer has been registered during annealing of the matrixes as well as in a series of experiments in which matrixes were deposited at increased substrate temperature. This allowed us to distinguish between the absorptions due to the two conformers.

The experiment is supported by theoretical predictions undertaken at different levels of approximation (MP2 and $\mathrm{DFT} / \mathrm{B} 3 \mathrm{LYP})$. It was shown that the syn conformer $(\mathrm{C}-\mathrm{C}-$ $\mathrm{C}=\mathrm{O}$ dihedral angle equal to $0^{\circ}$ ) corresponds to the conformational ground state, with the doubly degenerated by symmetry 
gauche conformer $(\mathrm{C}-\mathrm{C}-\mathrm{C}=\mathrm{O}$ dihedral angle equal to $\pm 139.9^{\circ}$ ) being only slightly less stable than the syn form $\left(\Delta E_{(\text {gauche-syn })}\right.$ in the gas phase is predicted by the MP2/6$31 \mathrm{G}^{* *}$ calculations as being $0.174 \mathrm{~kJ} \mathrm{~mol}^{-1}$ ). In the matrixes, the syn form is stabilized relative to the gauche conformer $\left(\Delta E_{\text {(gauche-syn) }} \approx 1.4 \mathrm{~kJ} \mathrm{~mol}^{-1}\right.$, in xenon). The predicted energy barriers for conformer interconversion were found to be significantly low: the MP2/6-31G** calculated $\Delta E_{(\text {syn } \rightarrow \text { gauche }}$ energy barrier is $c a$. $3.65 \mathrm{~kJ} \mathrm{~mol}^{-1}$, while the calculated energy barrier separating the two symmetrically equivalent gauche conformers stays only $0.196 \mathrm{~kJ} \mathrm{~mol}^{-1}$ above the energy of these forms. In the matrixes, $\Delta E_{(\text {syn } \rightarrow \text { gauche }}$ energy barrier was estimated to be $c a \cdot 17-18 \mathrm{~kJ} \mathrm{~mol}^{-1}$, while the $\Delta E_{\text {(gauche } \rightarrow \text { gauche })}$ energy barrier is, with all probability, still lower than in the gaseous phase. The matrix isolation spectroscopic data indicate that interconversion between the gauche conformers occurs even in the low temperature matrixes, resulting in the observed spectroscopic properties of the second conformational state resembling more those predicted for the anti structure than for the minimum energy gauche conformation.

\section{Acknowledgements}

The authors thank Prof. Ben van der Veken (RUCA Centre, University of Antwerp, Belgium) and Dr. Leszek Lapinski (Institute of Physics, PAN, Warsaw, Poland) for their helpful comments and suggestions and acknowledge the Portuguese Science Foundation (FCT) for financial support (Research projects PRAXIS/P/QUI/10137/1998, PRAXIS/PCEX/C/ QUI/108/96 and grant FCT \# SFRH/BPD/1661/2000).

\section{References}

1 J. M. F. Neta, Estudo Estrutural e Espectroscópico de Compostos Carbonílicos $\alpha$-ciano-substituídos, and references therein, Internal Report, Department of Chemistry, University of Coimbra, 1997 (Portuguese).

2 S. W. Charles, G. I. L. Jones and N. L. Owen, J. Chem. Soc., Faraday Trans. 2, 1973, 69, 1454.

3 D. Sinha and J. E. Katon, Can. J. Chem., 1974, 52, 3057.

4 S. J. Leibowitz, J. Laane, C. Van Alsenoy and B. J. Van der Veken, J. Mol. Struct., 1991, 248, 251.

5 J. M. F. Neta and R. Fausto, J. Mol. Struct., 1998, 443, 41.

6 J. J. C. Teixeira-Dias and R. Fausto, J. Mol. Struct., 1986, 144, 199.

7 R. Fausto and J. J. C. Teixeira-Dias, J. Mol. Struct., 1986, 144 215; R. Fausto and J. J. C. Teixeira-Dias, J. Mol. Struct., 1986, 144, 225; R. Fausto and J. J. C. Teixeira-Dias, J. Mol. Struct., $1986,144,241$
8 R. Fausto and J. J. C. Teixeira-Dias, THEOCHEM, 1987, 150, 381.

9 I. D. Reva, S. Stepanian, L. Adamowicz and R. Fausto, J. Phys. Chem. A, 2001, 105, 4773.

10 Grams/32 AI (version 6.0), Galactic Industries Coorporation, Salem, NH USA, 2000.

11 GAUSSIAN 98, Revision A.7., M. J. Frisch, G. W. Trucks, H. B. Schlegel, G. E. Scuseria, M. A. Robb, J. R. Cheeseman, V. G. Zakrzewski, J. A. Montgomery, Jr., R. E. Stratmann, J. C. Burant, S. Dapprich, J. M. Millam, A. D. Daniels, K. N. Kudin, M. C Strain, O. Farkas, J. Tomasi, V. Barone, M. Cossi, R. Cammi, B Mennucci, C. Pomelli, C. Adamo, S. Clifford, J. Ochterski, G. A. Petersson, P. Y. Ayala, Q. Cui, K. Morokuma, D. K. Malick, A. D. Rabuck, K. Raghavachari, J. B. Foresman, J. Cioslowski, J. V. Ortiz, A. G. Baboul, B. B. Stefanov, G. Liu, A. Liashenko, P. Piskorz, I. Komaromi, R. Gomperts, R. L. Martin, D. J. Fox, T. Keith, M. A. Al-Laham, C. Y. Peng, A. Nanayakkara, C. Gonzalez, M. Challacombe, P. M. W. Gill, B. Johnson, W. Chen, M. W. Wong, J. L. Andres, C. Gonzalez, M. Head-Gordon, E. S. Replogle and J. A. Pople, Gaussian, Inc., Pittsburgh, PA, 1998.

12 A. D. Becke, Phys. Rev. B, 1988, 38, 3098.

13 C. Lee, W. Yang and R. G. Parr, Phys. Rev. B, 1988, 37, 785.

14 S. H. Vosko, L. Wilk and M. Nusair, Can. J. Phys., 1980, 58, 1200.

15 W. J. Hehre, R. Ditchefield and J. A. Pople, J. Chem. Phys., 1972, 56, 2257.

16 M. J. Frisch, J. A. Pople and J. S. Binkley, J. Chem. Phys., 1984, 80, 3265.

17 C. Peng, P. Y. Ayala, H. B. Schlegel and M. J. Frisch, J. Comput Chem., 1996, 17, 49

18 A. Kulbida, M. N. Ramos, M. Räsänen, J. Nieminen, O. Schrems and R. Fausto, J. Chem. Soc., Faraday Trans., 1995, 91, 1571.

19 E. M. S. Maçôas, R. Fausto, M. Pettersson, L. Khriachtchev and M. Räsänen, J. Phys. Chem. A, 2000, 104, 6956.

20 E. M. S. Maçôas, R. Fausto, J. Lundell, M. Pettersson, L. Khriachtchev and M. Räsänen, J. Phys. Chem. A, 2000, 104, 11725 .

21 A. J. Barnes, J. Mol. Struct., 1984, 113, 161.

22 A. Serrallach and R. Meyer, J. Mol. Spectrosc., 1976, 60, 246.

23 A. J. Barnes and G. C. Whittle, Proceedings of the 12th European Congress on Molecular Spectroscopy, Elsevier, Amsterdam, 1976 p. 373.

24 F. Molnár and B. Dick, Ber. Bunsen-Ges. Phys. Chem., 1995, 99, 422.

25 R. Fraenkel and Y. Haas, Chem. Phys., 1994, 186, 185.

26 R. Fraenkel, S. Kahana and Y. Haas, Ber. Bunsen-Ges. Phys. Chem., 1995, 99, 412.

27 L. A. Eriksson and A. Laaksonen, J. Chem. Phys., 1996, 105, 8195.

28 R. Fausto, A. Kulbida and O. Schrems, J. Chem. Soc., Faraday Trans., 1995, 91, 3755.

29 G. G. Sheina and A. Yu. Ivanov, Vestn. Moscow State Univ., Ser. 2, Chemistry, 1995, 36, 320, (Russian).

30 M. Kofranek, A. Karpfen and H. Lischka, Chem. Phys. Lett., 1992, 189, 281. 